\title{
PERFORMANCE ASSESSMENT OF GAUSSIAN PROCESS REGRESSION TO PREDICT THE BOND STRENGTH OF FRP SHEETS TO CONCRETE
}

\author{
Thuy Anh Nguyen*, Hai Bang Ly \\ University of Transport Technology, 54 Trieu Khuc, Thanh Xuan, Hanoi, Vietnam
}

\author{
ARTICLE INFO \\ TYPE: Research Article \\ Received: $12 / 01 / 2021$ \\ Revised: 29/03/2021 \\ Accepted: 02/04/2021 \\ Published online: 27/05/2021 \\ https://doi.org/10.47869/tcsj.72.4.2 \\ *Corresponding author \\ Email: anhnt@utt.edu.vn
}

\begin{abstract}
A Gaussian process regression (GPR) model for predicting the bond strength of FRP-to-concrete is proposed in this study. Published single-lap shear test specimens are used to predict the bond strength of externally bonded FRP systems adhered to concrete prisms. A database of 150 experimental results collected from published works is used for the training and testing phases of the proposed GPR model, containing 6 input parameters (width of concrete prism, concrete compressive strength, FRP thickness, FRP width, FRP length, and FRP modulus of elasticity). The output parameter of the prediction problem is bond strength. Three statistical indicators, namely the coefficient of determination, root mean square error (RMSE), and mean absolute error (MAE) are used to evaluate the performance of the proposed GPR model over 500 simulations. The results of this study indicate that the GPR provides an efficient alternative method for predicting the bond strength of FRP-to-concrete when compared to experimental results.
\end{abstract}

Keywords: bond strength; FRP-to-concrete; Gaussian process regression.

C 2021 University of Transport and Communications

\section{INTRODUCTION}

Currently, strengthening and repairing reinforced-concrete (RC) structures using externally bonded fiber-reinforced polymer (FRP) plates or sheets have become a widely accepted solution [1-3]. Several advantages of FRP materials, such as high strength and corrosion resistance, non-magnetic interference, and higher strength to weight ratio, lead to 
reduced self-weight of the strengthened RC structures in comparison with those that use conventional steel reinforcement. Moreover, the high fatigue resistance makes them a viable alternative material to reinforce seismically deficient structures and also structures suffering from corrosion-related problems [4]. The externally bonded FRP plates can also be used to improve confinement in the compression members and increase the moment capacity of flexural members [5]. Furthermore, the high versatility and constructability of bonding FRP plates outside of concrete structures offers many advantages in civil and transport infrastructure applications because the FRP plates can be easily linked to structures with any cross-section [6]. However, the efficiency of FRP depends significantly on the bonding mechanism between FRP and concrete, which is controlled by several parameters such as the mechanical properties of concrete, FRP thickness, FRP modulus of elasticity, FRP length and width, and some other factors such as skillful labor, well treated and undamaged concrete surface or the epoxy quality [4]. Bonding failure is the most common type of failure in RC structures reinforced with external FRP plates [7]. As a result, extensive research on this topic has been carried out. Many experimental studies have been performed to investigate the bond strength using the single-lap shear tests [7-9]. In addition, theoretical studies using fracture mechanics analysis [10-12], and finite element analysis [13,14], and the development of empirical models $[15,16]$ have been proposed to study the bond strength of FRP-to-concrete joints. However, experimental and theoretical studies have to use several assumptions, as well as many limitations depending on each particular case, thereby losing its generality.

Recently, the development and application of machine learning in the field of construction has been widely studied [17-21]. Taking advantage of the tested database, machine learning algorithms demonstrate the ability to simplify classical approaches, such as the method of testing or numerical simulation. Among machine learning algorithms, Gaussian Process Regression is an efficient and reliable learning approach for modeling complex and nonlinear function mappings [22, 23]. Accordingly, the objective of this study is to evaluate the capability of the Gaussian Process Regression for modeling the bond strength of FRP to concrete, based on a dataset of 150 experimental results collected in international journals.

\section{METHODS USED}

\subsection{Gaussian Regression Process (GPR)}

Gaussian process regression (GPR) is a nonparametric, Bayesian approach applied to regression problems. GPR has several advantages, working well on small datasets and having the ability to provide uncertainty measurements on the prediction values.

Given the training data set $D=\left\{\left(X_{i}, Y_{i}\right)\right\}_{i=1}^{N}$, where $\mathrm{N}$ is the training set's dimension, $X_{i} \in R^{d}$ represent for input data, $Y_{i} \in R$ is the corresponding output value. In data set $\mathrm{D}$, random variables corresponding to input data set $\left\{X_{i}\right\}_{i=1}^{N}$ compose set $\left\{f\left(X_{1}\right), f\left(X_{2}\right), \ldots, f\left(X_{N}\right)\right\}$ and are subjected to the joint Gaussian distribution. For the simplest case, the relation between the latent function $\mathrm{f}(\mathrm{X})$ and the observed target $\mathrm{Y}$ is:

$$
\begin{gathered}
Y=f(X)+\varepsilon ; \quad f(X)=X^{T} . \mathrm{w}, \\
\text { where } \mathrm{w}: \mathrm{N}\left(0, \Sigma_{P}\right) ; \varepsilon: N\left(0, \sigma_{n}^{2}\right)
\end{gathered}
$$

where w denotes the weight, $\varepsilon$ is the independent noise, $\sigma_{n}^{2}$ is the variance of the noise, $\Sigma_{\mathrm{P}}$ 
is covariance. The distribution in the Gaussian process is represented by a mean function, denoted as $m(X)$, and a covariance kernel function, denoted as $K\left(X, X^{\prime}\right)$ [24]:

$$
f(X): G P\left[m(X), K\left(X, X^{\prime}\right)\right]
$$

where $X$ and $X^{\prime} \in R^{d}$ are random numbers of random variables. For the basic GPR, $\mathrm{m}(\mathrm{x})$ is set to be zero, and formula (1) can be rewritten as:

$$
f(X): G P\left[0, K\left(X, X^{\prime}\right)\right]
$$

where $\mathrm{X}$ is the learning sample whose measure in the GP is the finite-dimensional distribution of the GP. As defined by the GP, the finite-dimensional distribution is a joint normal distribution as:

$$
\left[f\left(X_{1}\right), f\left(X_{2}\right), \ldots, f\left(X_{n}\right)\right]^{T}: N(m, K)
$$

The noise $\varepsilon$ is free from $\mathrm{f}(\mathrm{x})$, and it is subject to the Gaussian distribution. When $\mathrm{f}(\mathrm{x})$ is an object of the Gaussian distribution, $y$ is also subjected to the Gaussian distribution. Then, the prior distribution of the observed target value $\mathrm{Y}$ is inferred as:

$$
Y: N\left(0, K(X, X)+\sigma_{n}^{2} I\right)
$$

With given test sample points $\left(X^{*}, Y^{*}\right)$, The joint probability distribution of observed target value $\mathrm{Y}$ and prediction value $Y^{*}$ at test points is expressed as:

$$
\left[\begin{array}{c}
Y \\
Y^{*}
\end{array}\right]: N\left(0,\left[\begin{array}{cc}
K(X, X)+\sigma_{n}^{2} I & K\left(X, X^{*}\right) \\
K\left(X^{*}, X\right) & K\left(X^{*}, X^{*}\right)
\end{array}\right]\right)
$$

where $K(X, X)=\left(K_{i j}\right)$ is a positive defined symmetry matrix of size $N \times N, K_{i j}=K\left(X_{i}\right.$, $\left.X_{j}\right)$ are the elements in the matrix, respectively, to measure the correlation of $X_{i}$ and $X_{j} ; K(X$, $\left.X^{*}\right)$ is the matrix of covariance of the training set and the testing set.

Application the conditional distribution properties of the Gaussian distribution, an equation is proposed:

$$
p\left(Y^{*} \mid X, Y, X^{*}\right)=N\left(Y^{*} \mid \bar{Y}^{*}, \operatorname{cov}\left(Y^{*}\right)\right)
$$

where:

$$
\begin{gathered}
\bar{Y}^{*}=K\left(X, X^{*}\right)^{T}\left[K(X, X)+\sigma_{n}^{2} I\right]^{-1} Y \\
\operatorname{cov}\left(Y^{*}\right)=K\left(X^{*}, X^{*}\right)-K\left(X, X^{*}\right)^{T}\left[K(X, X)+\sigma_{n}^{2} I\right]^{-1} K\left(X, X^{*}\right)
\end{gathered}
$$

the mean value $\bar{Y}^{*}$ is the estimation value of $Y^{*} ; \operatorname{cov}\left(Y^{*}\right)$ is the variance matrix of test samples, which reflects the estimation value's reliability.

In the GPR, the covariance (Kernel) function is a critical factor, as it defines the similarity of the data, which has a significant impact on the prediction results [25]. In this study, the following five types of covariance functions are used for predicting the bond strength of FRP to concrete [26]: 
Squared Exponential:

$$
K\left(X_{i}, X_{j} \mid \theta\right)=\sigma_{f}^{2} \exp \left[-\frac{1}{2} \frac{\left(X_{i}-X_{j}\right)^{T}\left(X_{i}-X_{j}\right)}{\sigma_{l}^{2}}\right]
$$

Rational quadratic:

$$
K\left(X_{i}, X_{j} \mid \theta\right)=\sigma_{f}^{2}\left(1+\frac{r^{2}}{2 \alpha \sigma_{l}^{2}}\right)^{-\alpha}
$$

Matern52:

$$
K\left(X_{i}, X_{j} \mid \theta\right)=\sigma_{f}^{2}\left(1+\frac{\sqrt{5} r}{\sigma_{l}}+\frac{5 r^{2}}{3 \sigma_{l}^{2}}\right) \exp \left[-\frac{\sqrt{5} r}{\sigma_{l}}\right]
$$

Matern32:

$$
K\left(X_{i}, X_{j} \mid \theta\right)=\sigma_{f}^{2}\left(1+\frac{\sqrt{3} r}{\sigma_{l}}\right) \exp \left[-\frac{\sqrt{3} r}{\sigma_{l}}\right]
$$

Exponential:

$$
K\left(X_{i}, X_{j} \mid \theta\right)=\sigma_{f}^{2} \exp \left[-\frac{r}{\sigma_{l}}\right]
$$

where " $\mathrm{r}$ " is the Euclidean distance between variables $\mathrm{X}_{\mathrm{i}}$ and $\mathrm{X}_{\mathrm{j}}$ :

$$
r=\sqrt{\left(X_{i}-X_{j}\right)^{T}\left(X_{i}-X_{j}\right)}
$$

and $\sigma_{l}$ and $\sigma_{f}$ are the characteristic length scale and the signal standard deviation, respectively.

\subsection{Hyper-parameters selection}

In the above GPR, given the introduction of noise, the variance function contains additional parameters that are termed as "Hyper-parameters". Specifically, hyper-parameters in the Bayes method refer to parameters that control the distribution of model parameters, namely the parameters of a parameter [27]. Hyper-parameters corresponding to GPR include the following:

- The first hyper-parameter denotes the variance ${ }^{\sigma_{n}^{2}}$ of noise $\varepsilon$,

- The second hyper-parameter denotes the covariance $\Sigma \mathrm{P}$ of weight vector w,

- The last hyper-parameter corresponds to parameters $\left(\sigma, \sigma_{f}\right)$ that are included in the kernel function $\mathrm{K}(\cdot, \cdot)$.

The results of several studies indicate that the fitting accuracy and generalization ability of GPR improved via selecting optimal hyper-parameters [28, 29].

\subsection{Performance criteria}

In this study, to evaluate the accuracy of predictive results, three different assessment metrics, namely the coefficient of determination $\left(\mathrm{R}^{2}\right)$, root mean square error (RMSE), and mean absolute error (MAE) are utilized to compute the prediction errors of the proposed 
model. The formulations are listed in equations (16) - (18), respectively.

$$
\begin{gathered}
R^{2}=1-\frac{\sum_{j=1}^{N}\left(q_{j}-q_{j}\right)^{2}}{\sum_{j=1}^{N}\left(q_{j}-\bar{q}_{j}\right)^{2}} \\
R M S E=\sqrt{\frac{1}{N} \sum_{j=1}^{N}\left(q_{j}-q_{j}\right)^{2}} \\
M A E=\frac{1}{N} \sum_{j=1}^{N}\left|q_{j}-q_{j}\right|
\end{gathered}
$$

where $\mathrm{N}$ is the number of samples, $q_{j}$ is the actual value; $\stackrel{q}{q}_{j}$ is the predicted value; $\bar{q}_{j}$ is the average of actual values.

\section{DATABASE CONSTRUCTION}

To build a predictive model of the bond strength of FRP-to-concrete, a database of 150 test results is collected from the various documents [7-9, 30-34]. The input variables affecting the bonding force considered in this study include the width of concrete prism $\left(\mathrm{I}_{1}\right)$, concrete cylinder compressive strength $\left(\mathrm{I}_{2}\right)$, the width of FRP $\left(\mathrm{I}_{3}\right)$, the thickness of FRP $\left(\mathrm{I}_{4}\right)$, the elastic modulus of FRP $\left(\mathrm{I}_{5}\right)$, and bond length $\left(\mathrm{I}_{6}\right)$. In the collected database, the value of the width of concrete prism varies in the range of $100-228.2 \mathrm{~mm}$, the concrete cylinder compressive strength is in the range $16-50 \mathrm{MPa}$, the width of FRP varies from $10-39.9 \mathrm{~mm}$, the thickness of FRP ranges between 0.08 and $0.84 \mathrm{~mm}$, the elastic modulus of FRP value varies from 83.03 to $300 \mathrm{MPa}$, and the bond length ranges from $50-150.6 \mathrm{~mm}$. Besides, the bond strength of FRP-to-concrete values are in the range of $4.11-46.35 \mathrm{kN}$. The quantitative analysis of input and output parameters is detailed in Table 1. All the results are single-lap shear tests, and the beam test diagram is illustrated in Fig. 1. Among all the specimens in the database, $105(70 \%)$ are randomly selected, used as training sets, and the remaining 45 samples (30\%) are used to investigate the accuracy of the trained GPR model.

Table 1. Statistical analysis of the input and output variables used in this study.

\begin{tabular}{|c|c|c|c|c|c|c|}
\hline Variable & Min & Median & Mean & Max & $\begin{array}{c}\text { Standard } \\
\text { deviation }\end{array}$ & Skewness \\
\hline $\mathbf{I}_{1}$ & 100.000 & 150.000 & 161.348 & 228.200 & 40.633 & -0.238 \\
\hline $\mathbf{I}_{2}$ & 16.000 & 30.000 & 33.677 & 50.000 & 9.298 & 0.295 \\
\hline $\mathbf{I}_{3}$ & 10.000 & 40.000 & 39.891 & 100.000 & 21.542 & 0.875 \\
\hline $\mathbf{I}_{4}$ & 0.080 & 1.020 & 0.840 & 1.400 & 0.534 & -0.182 \\
\hline $\mathbf{I}_{5}$ & 83.030 & 152.200 & 177.995 & 300.000 & 58.554 & 0.132 \\
\hline $\mathbf{I}_{6}$ & 50.000 & 150.000 & 150.593 & 300.000 & 70.919 & 0.718 \\
\hline $\mathbf{Y}$ & 4.110 & 11.240 & 14.784 & 46.350 & 9.866 & 1.352 \\
\hline
\end{tabular}



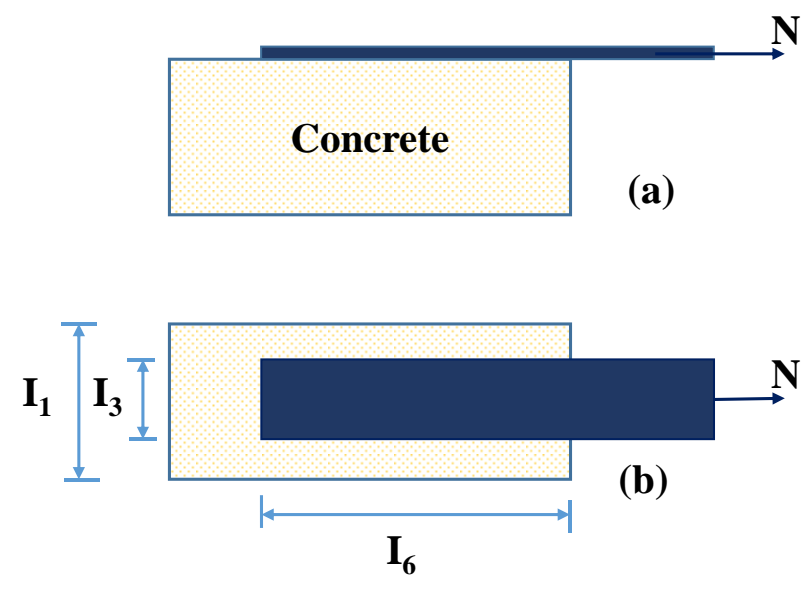

Figure 1. Test specimen (a) side view (b) top view.

The correlations between the inputs and bond strength of FRP-to-concrete is shown in Fig. 2. The correlation values are represented by different colors. It is observed that the correlations between the inputs and output are not strictly linear, with a maximum correlation value of about 0.5 . So that all input variables are used to construct the GPR model.

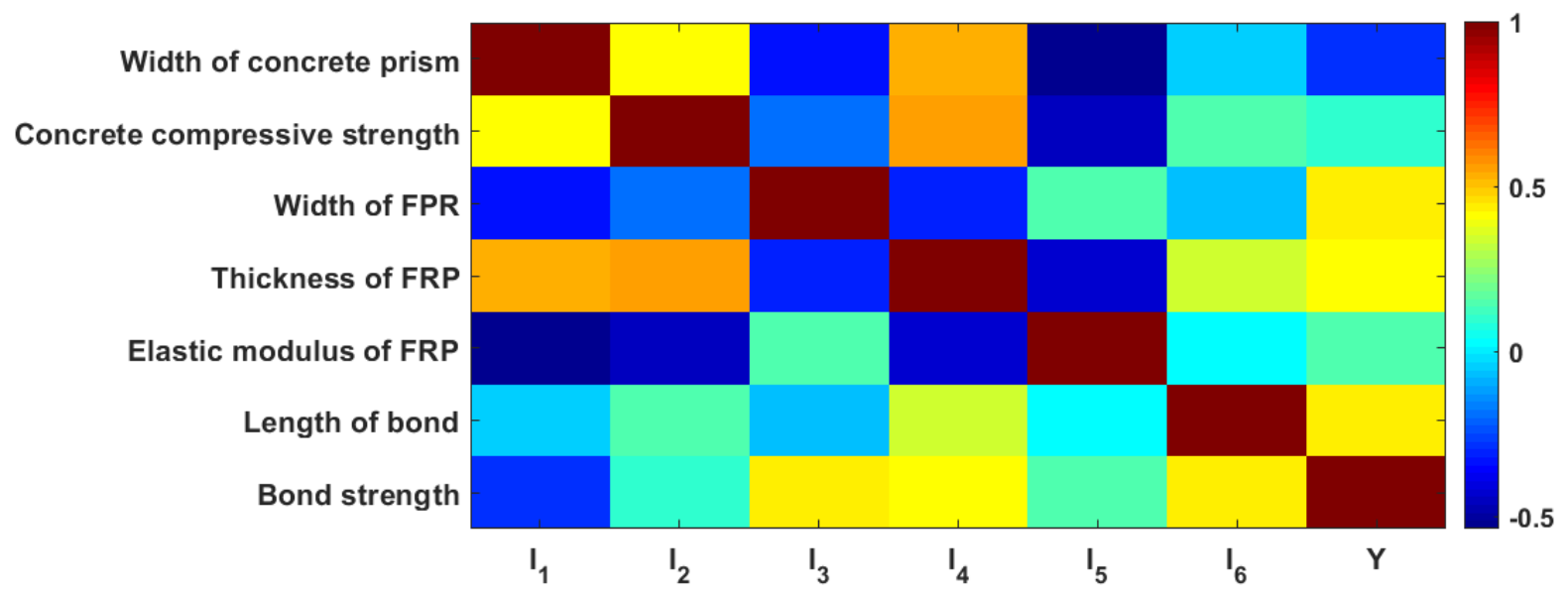

Figure 2. Multi-correlation graph of input and output variables used in this study.

\section{RESULTS AND DISCUSSION}

\subsection{GPR prediction capability}

GPR model is used in this study to predict the bond strength. As mentioned above, the selection of hyper-parameters is crucial to obtain reliable and high accuracy outputs. Besides, the accuracy of GPR, or any machine learning algorithms, greatly depends on the selection process of the samples in the training dataset. Therefore, 500 simulations taking into account the random sampling effect, which randomly select $70 \%$ of the total data to generate the training dataset, are performed to fully evaluate the performance of the proposed GPR. During the simulation process, the hyper-parameters are automatically optimized and selected using Bayesian optimization by minimizing the out-of-sample mean square error in function of different cross-validation values.

Fig. 3 shows the results of RMSE, MAE and $\mathrm{R}^{2}$ of the testing parts for a total of 500 
simulations. It can be seen that the GPR model, using automatic hyper-parameters optimization, gives excellent prediction results. Indeed, the RMSE values of bond strength range mainly in the 2-3 $(\mathrm{kN})$ range, the MAE values of bond strength range in the 1-2 $(\mathrm{kN})$ range, whereas the $R^{2}$ values are mostly found in the 0.93 to 0.99 range.

Fig. 4a shows the results of the hyper-parameters selected by the Bayesian optimization process over 500 simulations. It can be seen that the kernel matern32 (Matern kernel with parameter 3/2) functions are mostly selected, following by the exponential kernel, the rational quadratic kernel function, kernel matern52, and squared exponential function. It is worth noticing that the ARD denotes that the use of the corresponding function but using a separate length scale per predictor. Fig. $4 \mathrm{~b}$ shows the basis functions used in the simulation, where the pure quadratic function is mostly selected by the Bayesian optimization process, following by the choice that no basis function is used, linear function, and a constant as basis function. Finally, a small value (around 0) of sigma is preferred, whereas several values in the range of 1 to 20 are selected by the optimization process (Fig. 4c).

Finally, the statistical results of error indicators are presented in Table 2 for the training and testing datasets.

(a)

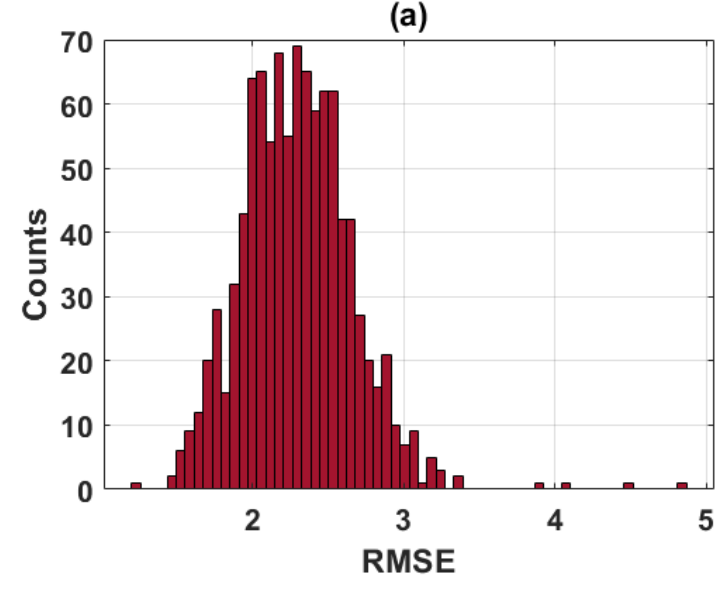

(b)

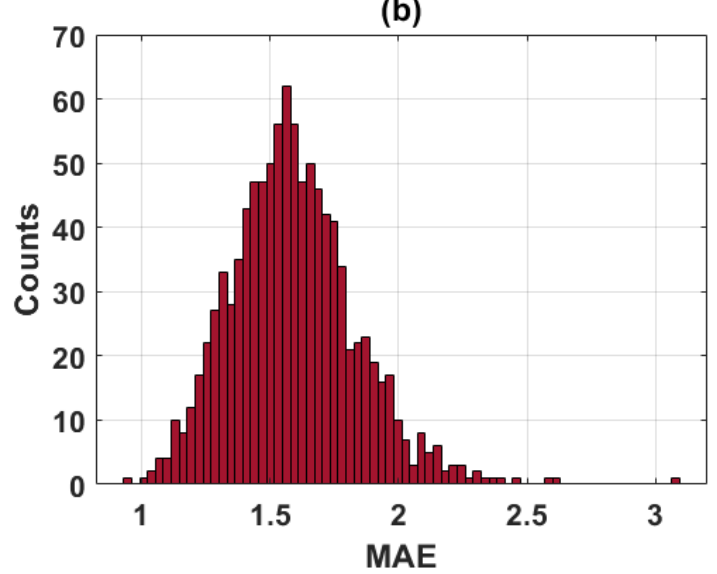

(c)

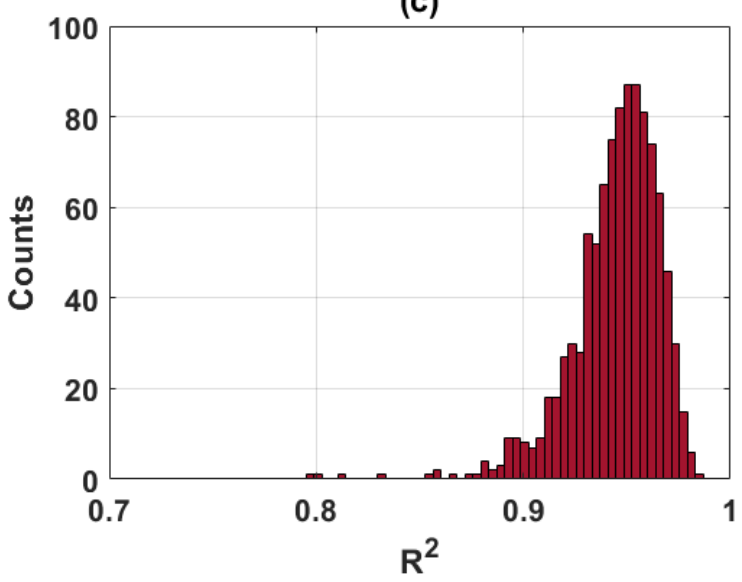

Figure 3. A graph showing the best testing performance of GPR over 500 simulations, taking into account the random sampling effect for (a) RMSE, (b) MAE, and (c) $\mathrm{R}^{2}$. 
Transport and Communications Science Journal, Vol. 72, Issue 4 (05/2021), 411-422

(a) Kernel Function

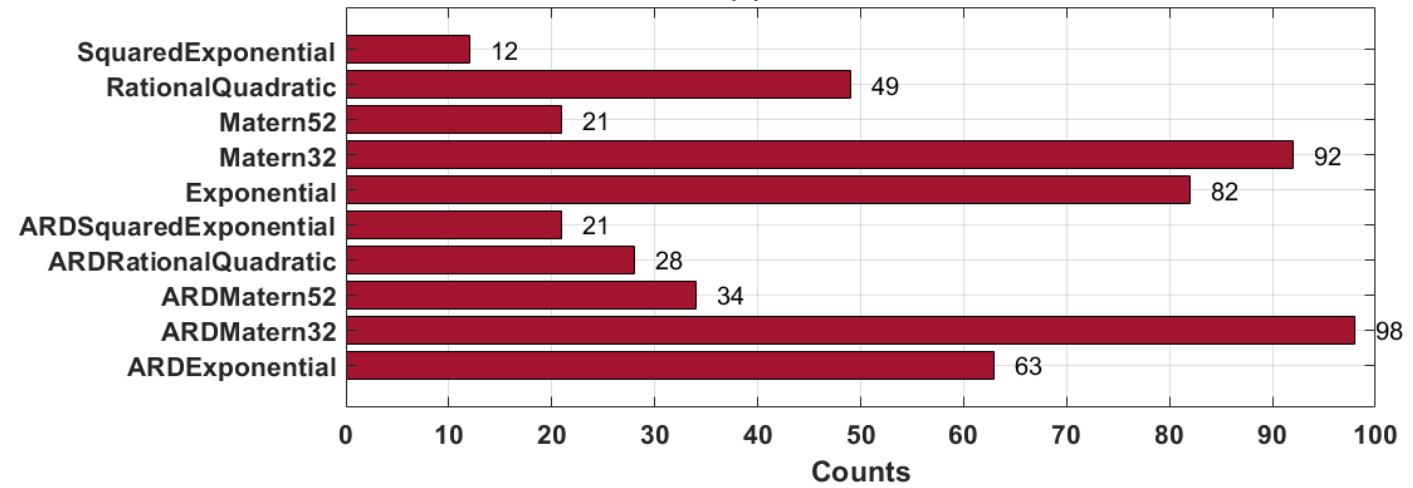

(b) Basis Function

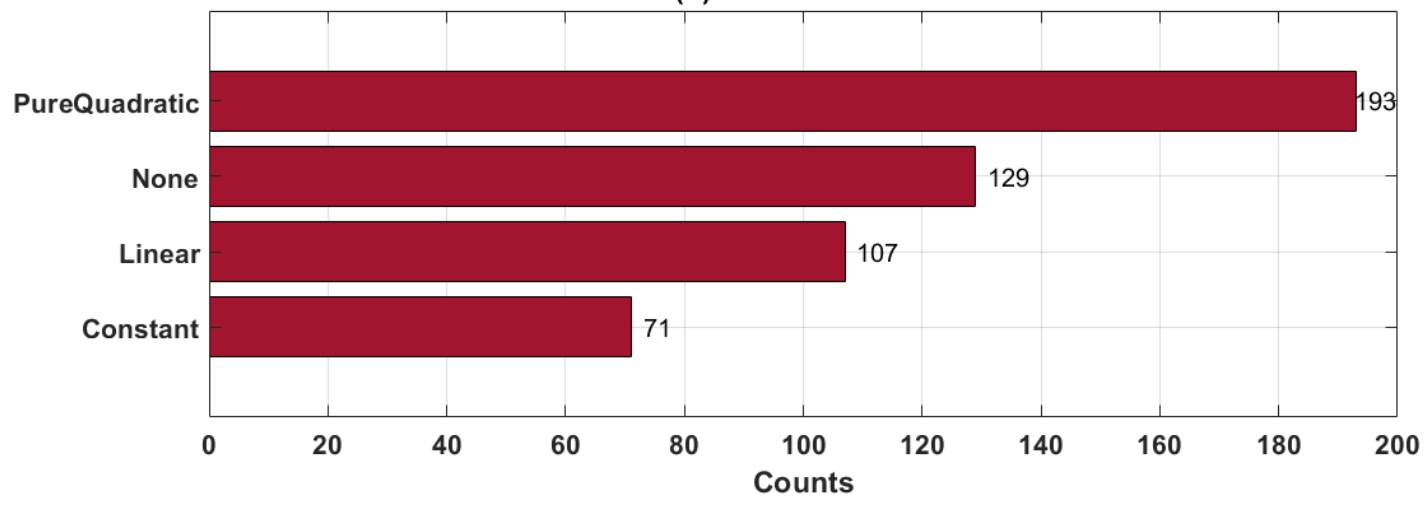

(c) Sigma values

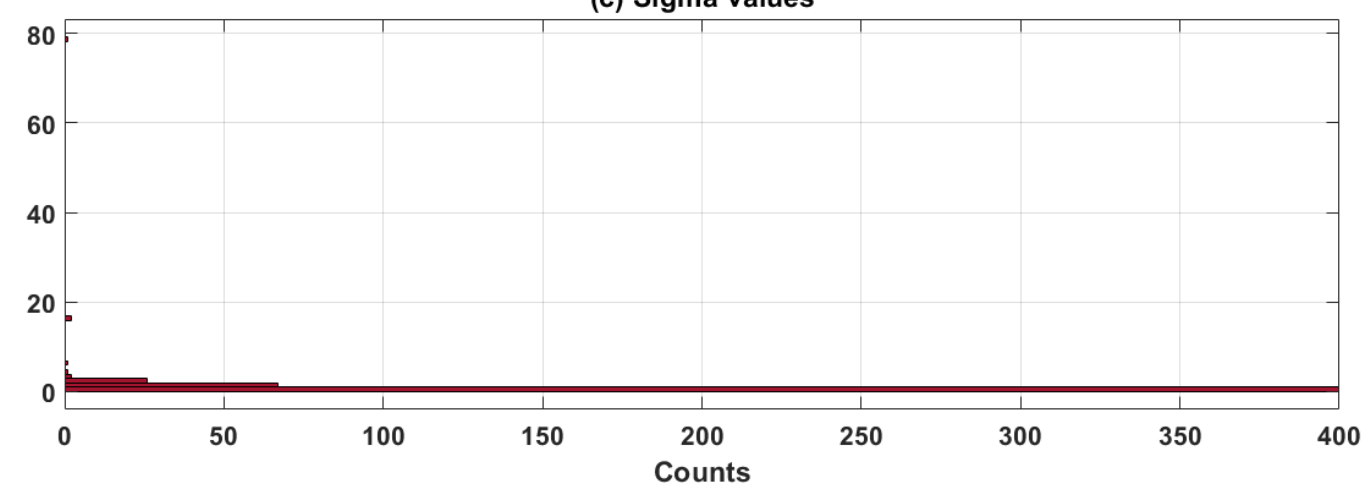

Figure 4. A graph showing the GPR hyper-parameters selection results for (a) kernel function, (b) basis function, and (c) sigma values.

Table 2. Statistical analysis results of $\mathrm{R}^{2}$, RMSE, and MAE distributions over 500 runs.

\begin{tabular}{lccc}
\hline \hline & RMSE & MAE & $\mathbf{R}^{\mathbf{2}}$ \\
\hline Training dataset & & & \\
\hline Max & 3.333 & 2.538 & 0.974 \\
\hline Average & 2.340 & 1.657 & 0.942 \\
\hline Min & 1.596 & 1.130 & 0.890 \\
\hline Testing dataset & & & \\
Max & 3.493 & 2.568 & 0.985 \\
\hline Average & 2.306 & 1.643 & 0.945 \\
\hline Min & 1.250 & 0.901 & 0.882 \\
\hline
\end{tabular}




\subsection{GPR prediction performance}

In this section, the prediction results of GRP with the best predictive capacity over 500 simulations are presented. Fig. 5a, b shows a strong correlation between predicted and actual bond strength values for the training and testing datasets through regression graphs, respectively. A linear fit is applied and plotted in each case. Besides, most of the predicted values of the training, testing datasets are close to the $95 \%$ confidence bounds. The values of the coefficient of determination are 0.934 and 0.985 for the training and testing datasets, respectively. It could be concluded that predicting the bond strength FRP-to-concrete is possible using the GPR model.

(a)

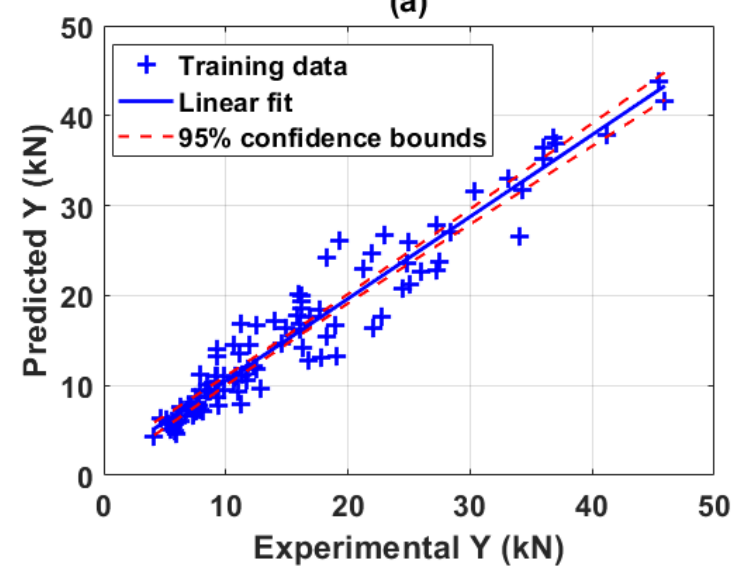

(b)

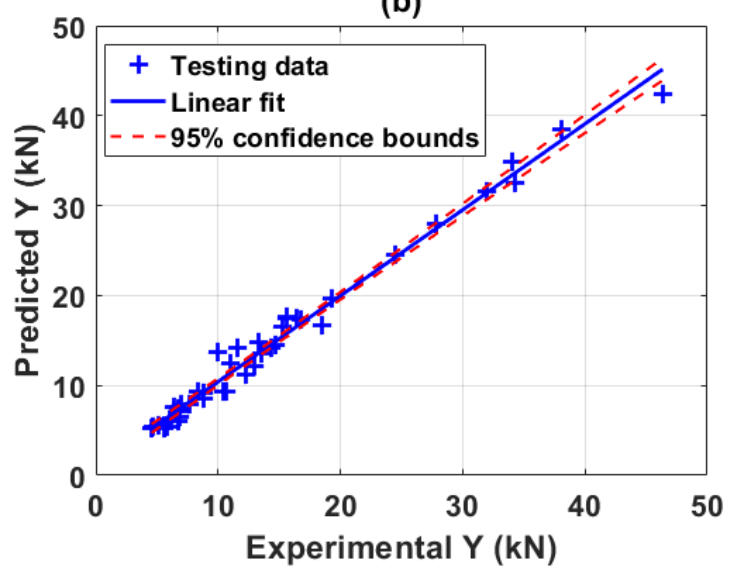

Figure 5. Correlation results of actual and predicted bond strength values for the training dataset (a) and testing dataset (b) of GPR.

The comparison between the experimental and predicted values of bond strength by GPR algorithm is shown in Fig. 6 for the training and testing datasets. The calculated values of RMSE are 2.538 and $1.25(\mathrm{kN})$ for the training and testing datasets, respectively. Besides, the values of MAE are 1.842 and $0.901(\mathrm{kN})$ for training and testing datasets in this case, respectively. These results show that the predicted values are very close to the experimental ones, showing the significant performance of GPR in predicting the bond strength of FRP-toconcrete.

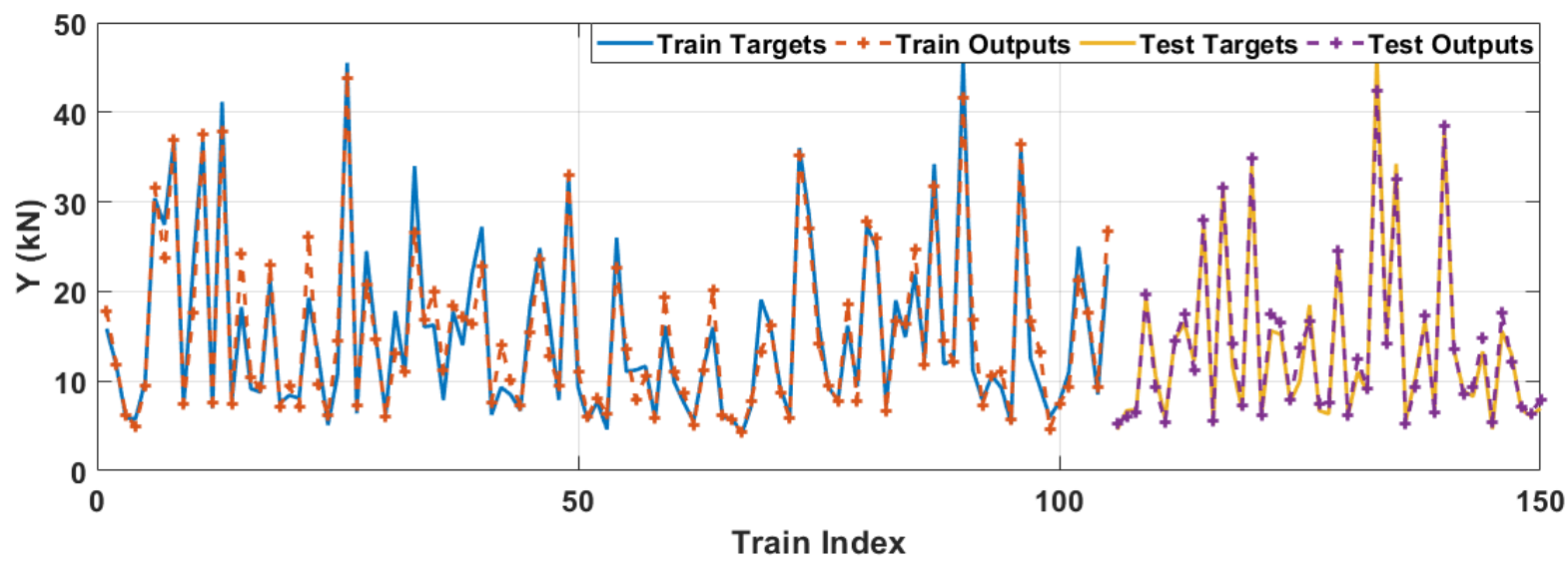

Figure 6. Comparison of the predicted and actual results for the training and testing datasets. 
The error histogram of the GPR algorithm is plotted for the training dataset (Fig. 7a) and testing dataset (Fig. 7b). The error values corresponding to the training and testing datasets are small, in general. Besides, based on the cumulative distribution (red line), the percentage error of the samples within a range can be easily determined. For example, with the training dataset, the percentage of samples with error in the range $[-2 ; 2] \mathrm{kN}$ is about $80 \%$. Similarly, the $90 \%$ error between the experimental value and the GPR simulation of the testing data is about $[-2 ; 2] \mathrm{kN}$. These error percentages show that the GPR algorithm is an excellent choice for quick estimation of the bond strength of FRP-to-concrete.

(a)

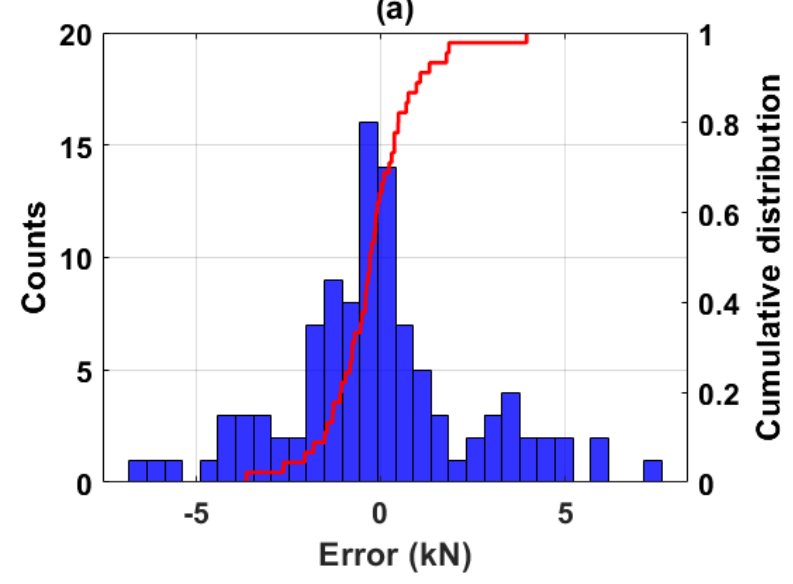

(b)

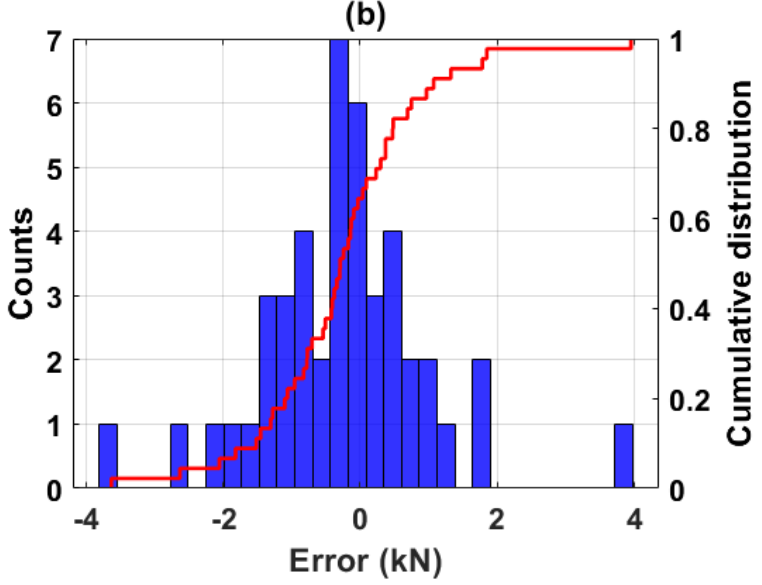

Figure 7. Error for the training and testing datasets.

\section{CONCLUSION}

Gaussian process regression (GPR) is used in this research to develop a model that could predict the bond strength of FRP-to-concrete under direct pull-out tension. The GPR model is constructed using a database containing 150 test specimens, collected from the reliable literature. The model is developed using six input parameters that predominantly control the bond strength of FRP-to-concrete. These parameters are the width of concrete prism, concrete cylinder compressive strength, the width of FRP, the thickness of FRP, elastic modulus of FRP, and bond length. The performance evaluation of GPR model is carried on by common statistical measurements such as $\mathrm{R}^{2}$, RMSE, and MAE. This model shows high reliability in predicting the bond strength $\left(\mathrm{R}^{2}=0.985, \mathrm{RMSE}=1.250\right.$, and MAE $\left.=0.901\right)$. The results of this study can be used to quickly and accurately predict the bond strength of FRT-to-concrete, useful for engineers in the calculation and design processes.

\section{REFERENCES}

[1] D. Oehlers, R. Seracino, Design of FRP and steel plated RC structures: retrofitting beams and slabs for strength, stiffness and ductility, Elsevier, 2004.

[2] J. G. Teng, J. F. Chen, S. T. Smith, L. Lam, FRP: strengthened RC structures, 2002.

[3] J. G. Teng et al., Behaviour and strength of FRP-strengthened RC structures: a state-of-the-art review, Proceedings of the Institution of Civil Engineers-Structures and Buildings, 156 (2003) 51-62. https://doi.org/10.1680/stbu.2003.156.1.51

[4] Y. Murad, A. Ashteyat, R. Hunaifat, Predictive model to the bond strength of FRP-to-concrete under direct pullout using Gene expression programming, Journal of Civil Engineering and Management, 25 (2019) 773-784. https://doi.org/10.3846/jcem.2019.10798 
[5] S. M. Hamze-Ziabari, A. Yasavoli, Predicting Bond Strength between FRP Plates and Concrete Sub-strate: Applications of GMDH and MNLR Approaches, Journal of Advanced Concrete Technology, 15 (2017) 644-661. https://doi.org/10.3151/jact.15.644

[6] Z. S. Wu, H. Yoshizawa, Analytical/experimental study on composite behavior in strengthening structures with bonded carbon fiber sheets, Journal of Reinforced Plastics and Composites, 18 (1999) 1131-1155. https://doi.org/10.1177/073168449901801207

[7] J. Yao, J. G. Teng, J. F. Chen, Experimental study on FRP-to-concrete bonded joints, Composites Part B: Engineering, 36 (2005) 99-113. https://doi.org/10.1016/j.compositesb.2004.06.001

[8] M. J. Chajes, W. W. Finch, T. A. Thomson, Bond and force transfer of composite-material plates bonded to concrete, Structural Journal, 93 (1996) 209-217. https://doi.org/10.14359/1491

[9] S. K. Sharma et al., Plate-concrete interfacial bond strength of FRP and metallic plated concrete

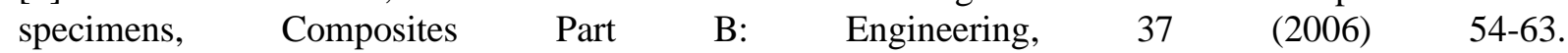
https://doi.org/10.1016/j.compositesb.2005.05.011

[10] H. YUAN, Z. WU, H. YOSHIZAWA, Theoretical solutions on interfacial stress transfer of externally bonded steel/composite laminates, Doboku Gakkai Ronbunshu, 2001 (2001) 2739. https://doi.org/10.2208/jscej.2001.675_27

[11] Z. Wu, H. Yuan, H. Niu, Stress transfer and fracture propagation in different kinds of adhesive $\begin{array}{lllll}\text { joints, Journal of Engineering } & \text { 5echanics, } & 128 & \text { (2002) }\end{array}$ https://doi.org/10.1061/(ASCE)07339399(2002)128:5(562)

[12] H. Yuan et al., Full-range behavior of FRP-to-concrete bonded joints, Engineering Structures, 26 (2004) 553-565. https://doi.org/10.1016/j.engstruct.2003.11.006

[13] J. F. Chen, Z. J. Yang, G. D. Holt, FRP or steel plate-to-concrete bonded joints: effect of test methods on experimental bond strength, Steel and Composite Structures, 1 (2001) 231-244. https://doi.org/10.12989/scs.2001.1.2.231

[14] H. Niu, Z. Wu, Interfacial debonding mechanism influenced by flexural cracks in FRPstrengthened beams, Journal of Structural Engineering, 47 (2001) 1277-1288.

[15] J. F. Chen, J. G. Teng, Anchorage strength models for FRP and steel plates bonded to concrete, Journal of Structural Engineering, 127 (2001) 784-791. http://hdl.handle.net/10397/29977

[16] O. Chaallal, M. -J. Nollet, D. Perraton, Strengthening of reinforced concrete beams with externally bonded fiber-reinforced-plastic plates: design guidelines for shear and flexure, Canadian Journal of Civil Engineering, 25 (1998) 692-704. https://doi.org/10.1139/198-008

[17] T. A. Nguyen, H. B. Ly, Development of ANN-based models to predict the bond strength of GFRP bars and concrete beams, Transport and Communications Science Journal, 71 (2020) 814-827. https://doi.org/10.47869/tcsj.71.7.7

[18] H. Q. Nguyen et al., Optimization of Artificial Intelligence System by Evolutionary Algorithm for Prediction of Axial Capacity of Rectangular Concrete Filled Steel Tubes under Compression, Materials, 13 (2020) 1205. https://doi.org/10.3390/ma13051205

[19] T. A. Nguyen, H. B. Ly, Prediction of critical elastic buckling load of cellular h-section beams using support vector machine, Transport and Communications Science Journal, 71 (2020) 500-513. https://doi.org/10.25073/tcsj.71.5.4

[20] H. B, Ly, T. A. Nguyen, Artificial neural network based modeling of the axial capacity of rectangular concrete filled steel tubes, Transport and Communications Science Journal, 71 (2020) 154166. https://doi.org/ 10.25073/tcsj.71.2.10

[21] D.V. Dao et al., Investigation and Optimization of the C-ANN Structure in Predicting the Compressive Strength of Foamed Concrete, Materials, $13 \quad$ (2020) 1072. https://doi.org/10.3390/ma13051072

[22] J. Hu, J. Wang, Short-term wind speed prediction using empirical wavelet transform and Gaussian process regression, Energy, 93 (2015) 1456-1466. 
Transport and Communications Science Journal, Vol. 72, Issue 4 (05/2021), 411-422

https://doi.org/10.1016/j.energy.2015.10.041

[23] L. Zhou, J. Chen, Z. Song, Recursive Gaussian process regression model for adaptive quality monitoring in batch processes, Mathematical Problems in Engineering, 2015 (2015) 9. https://doi.org/10.1155/2015/761280

[24] S. Banerjee et al., Gaussian predictive process models for large spatial data sets, Journal of the Royal Statistical Society: Series B (Statistical Methodology). 70 (2008) 825-848. https://doi.org/10.1111/j.1467-9868.2008.00663

[25] C. E. Rasmussen, Gaussian processes in machine learning, in: Summer School on Machine Learning, Springer, (2003), pp. 63-71. http://www.tuebingen.mpg.de/ carl

[26] I. Murray, R. P. Adams, Slice sampling covariance hyperparameters of latent Gaussian models, Advances in neural information processing systems, (2010), pp. 1732-1740.

[27] S. Sundararajan, S. S. Keerthi, Predictive approaches for choosing hyperparameters in Gaussian processes, Neural Computation. 13 (2001) 1103-1118. https://doi.org/10.1162/08997660151134343

[28] C. Xu et al., Intelligent analysis model of landslide displacement time series based on coupling PSO-GPR, Rock and Soil Mechanics, 32 (2011) 1669-1675.

[29] K. Liu, B. Liu, C. Xu, Intelligent analysis model of slope nonlinear displacement time series based on genetic-Gaussian process regression algorithm of combined kernel function, Chinese Journal

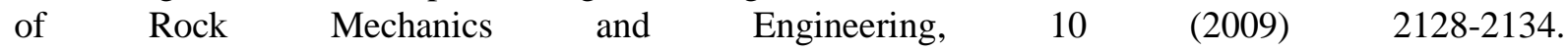
http://www.rockmech.org/EN/Y2009/V28/I10/2128

[30] K. Takeo et al., Bond characteristics of CFRP sheets in the CFRP bonding technique, Proceedings of Japan Concrete Institute, 19 (1997) 1599-1604.

[31] S. -K. Woo, Y. Lee, Experimental study on interfacial behavior of CFRP-bonded concrete, KSCE Journal of Civil Engineering, 14 (2010) 385-393. https://doi.org/10.18770/KEPCO.2015.01.01.127

[32] H. Toutanji, P. Saxena, L. Zhao, T. Ooi, Prediction of interfacial bond failure of FRP-concrete surface, Journal of Composites for Construction, 11 (2007) 427-436. http://worldcat.org/issn/10900268

[33] H. D. Zhao, Y. Zhang, M. Zhao, Research on the bond performance between CFRP plate and concrete, in Proc., 1st Conf. on FRP Concrete Structures of China, (2000), pp. 247-253.

[34] H. T. Ren, Study on basic theories and long time behavior of concrete structures strengthened by fiber reinforced polymers, Dalian University of technology, 2003. 